\title{
EL PAPEL DE LAALFABETIZACIÓN DIGITAL EN LA EMPLEABILIDAD DE LOS TRABAJADORES MAYORES
}

\section{THE ROLE OF DIGITAL LITERACY IN THE EMPLOYABILITY OF OLDER WORKERS}

\author{
Dra. Ma José Gómez Torres \\ gomeztor@us.es \\ Universidad de Sevilla. Facultad de Ciencias de la Educación. \\ Departamento de Didáctica y Organización Educativa. \\ C/ Pirotecnia, s/n, 41013, Sevilla (España)
}

Con la llegada del anunciado envejecimiento de la población, los conceptos de envejecimiento activo y formación permanente son parte de las agendas de los países miembros de la Unión Europea en la lucha contra el desempleo que sufren los trabajadores mayores de 55 años. Este estudio presenta una parte de los resultados obtenidos en el análisis de las transiciones laborales por las que pasan los trabajadores mayores en la actualidad; prestando especial atención a las relaciones que existen entre el nivel de alfabetización digital que poseen y su nivel de empleabilidad dentro del mercado de trabajo.

Palabras clave: Aprendizaje a lo largo de toda la vida, trabajadores mayores, alfabetización digital, educación y empleo.

Announced the arrival of an aging population, the concepts of active ageing and lifelong learning are part of the agendas of the member countries of the European Union in the fight against unemployment suffered by workers over 55 years old. This research presents some of the results obtained in the analysis of labor transitions that the older workers living today; it gives special attention to the relationships between the level of digital literacy that they own and their level of employability in the labor market.

Keywords: Lifelong learning, older workers, digital literacy, education and employment. 


\section{Introducción.}

En la actualidad la tasa de desempleo juvenil en España sigue siendo la más alta de la Unión Europea (UE), al mismo tiempo que la correspondiente al desempleo de las personas mayores continúa su ascenso imparable año a año. Son dos sectores de la población que representan realidades muy distintas acuciadas por el mismo problema: su escaso índice de empleabilidad dentro del mercado de trabajo.

Determinar el índice de empleabilidad que posee una persona no resulta fácil porque el término empleabilidad es un concepto difícil de cuantificar y medir, ya que siempre está ligado al puesto de trabajo concreto al que se accede o se pretende acceder. De esta manera, una persona puede disfrutar al mismo tiempo de una empleabilidad diferente en función de las ofertas de empleo a las que aspire en un momento dado o, por el contrario, carecer absolutamente de opciones debido a la falta de las competencias necesarias y suficientes para tener el éxito deseado dentro del mercado laboral (Gómez \& Ordóñez, 2014). Por lo tanto, cuando el empleo escasea la formación ofrece a las personas la posibilidad de adquirir las competencias de empleabilidad que les permitirán salir de la posición de desventaja en la que se encuentran, y obtener:

el conjunto de capacidades esenciales para aprender y desarrollarse eficazmente en el puesto de trabajo; incluyendo capacidades de comunicación y relación interpersonal, de resolución de problemas y manejo de procesos organizacionales, y de organización de los propios comportamientos en función de los requerimientos del puesto de trabajo (Marhuenda, Bernard \& Navas, 2010, p.143).

Los datos muestran que cuanto mayor es el nivel formativo más se reduce el riesgo de caer en la pobreza de competencias, es decir, el principal factor que obstaculiza las aspiraciones de inserción en el mercado de trabajo de los trabajadores mayores complicando sus transiciones laborales, sobradamente condicionadas ya por las demandas de la economía global. La situación actual ha propiciado que los itinerarios, o transiciones laborales, tradicionalmente caracterizados por constituir patrones secuenciales, universales y generalizados en las vidas de las personas, hayan dejado de ser lineales para pasar a ser discontinuos como consecuencia de diversos factores entre los que se encuentran: la precariedad laboral, los contratos de poca duración, las facilidades para el despido y la incertidumbre que rodea el mercado laboral.

Puesta en evidencia la estrecha relación que existe entre formación y empleo queda justificada, así, la presente aportación, ubicada dentro de las Ciencias de la Educación, en el proyecto I+D+i denominado DER 2012-36755 «Mercado de trabajo, transiciones laborales y edad: jóvenes y mayores de años» que persigue analizar las transiciones laborales de los dos segmentos de la población más castigados por la crisis financiera actual: los jóvenes y las personas mayores de 55 años de edad.

En esta ocasión, nuestro objetivo se ha centrado en el estudio de las condiciones en las que realizan en la actualidad las transiciones laborales de las personas mayores de 55 años en España, dentro del marco de las iniciativas desarrolladas por la UE en su lucha contra las altas tasas de desempleo que castiga a este colectivo, especialmente en nuestro país. Dentro del ámbito formativo se ha prestado especial atención a la brecha digital que sufren las personas y trabajadores mayores (más 
acusada aún en el caso de las mujeres) con el análisis de las medidas desarrolladas para garantizar una correcta y eficaz alfabetización digital entre los trabajadores mayores, tan necesaria para afrontar la inclusión social plena en la sociedad del conocimiento. En última instancia subyace la intención de ofrecer más información que permita conocer las claves que posibiliten una formación permanente orientada al empleo, individualizada y acorde con las necesidades de los ciudadanos, sea cual sea su edad.

\section{Método.}

Para el estudio del fenómeno que nos ocupa se han analizado más de un centenar de fuentes documentales tanto primarias como secundarias. La metodología utilizada se inscribe en el denominado análisis de documentos, dado que constituye un proceso enfocado al estudio de los hechos, decisiones y fenómenos contenidos en un soporte fácilmente recuperable, material y virtual, que representan la manifestación de la realidad social que recogen (López, 2002). Dentro del estudio de la realidad social los documentos de carácter educativo y formativo constituyen una parte esencial para comprender el fenómeno que nos ocupa en el presente trabajo, que gira en torno a los siguientes objetivos:

a) Conocer las características de las personas mayores en España.

b) Conocer el contexto socio-laboral actual y su impacto en las personas mayores.

c) Conocer el nivel de alfabetización digital de los trabajadores mayores como indicador básico de empleabilidad en el mercado laboral.

La recogida de los datos se ha llevado a cabo mediante una escala de observación como medio para obtener la información necesaria a través de los documentos. Para su elaboración realizamos una revisión bibliográfica exhaustiva y elaboramos un banco de datos sobre el que hemos trabajado en base a las siguientes categorías:

Trabajadores mayores en España. Esta categoría recoge seis indicadores relacionados con: mercado de trabajo de personas mayores de 55 años; principios de envejecimiento activo; personas activas; mejora de calidad de vida en la vejez; formación permanente y aprendizaje permanente.

- Contexto socio-laboral. Incluimos en esta categoría siete indicadores relacionados con: jubilación anticipada; sistema de pensiones; organización del trabajo; estrategias de formación en TIC; acuerdos de la UE en el empleo; políticas de empleo a nivel comunitario, nacional y local, y la valoración del impacto de la iniciativa española frente a la crisis en el empleo.

Alfabetización digital. Analizamos esta categoría a través de los siguientes indicadores: capacidad de aprendizaje; potencial productivo; rendimiento de las personas mayores; retos a los que se enfrentan; dominio de lenguajes tecnológicos y audiovisuales; brecha digital; condición de persona mayor; esperanza de vida y situación de exclusión social.

Para la validación de la escala se ha utilizado la metodología de juicio de expertos, para lo que se elaboró un protocolo anexo a la escala en el que diferentes expertos, en una escala de valoración de 1 a 5 , asignaban una puntuación considerando si los indicadores de cada categoría se adecuaban o no a los objetivos propuestos (Outón \& Suárez, 2010). A través del análisis se ha podido constatar que las categorías que configuran la escala 
tienen un nivel elevado de validez, dado que los indicadores han obtenido un nivel de puntuación alto para certificar su adecuación a los objetivos propuestos.

Para el análisis de datos cualitativos se ha utilizado el programa Atlas-ti que ha facilitado la tarea de establecer relaciones entre los datos obtenidos de las fuentes documentales ofreciendo una "potente e intuitiva red de comentarios y categorías que permiten mantener los datos bajo análisis permanente» (Blanco, 2012, p.107).

Los resultados que se aportan en este trabajo son solo una parte de los obtenidos tras el análisis de contenido realizado a las bases documentales, básicamente de carácter normativo, académico y estadístico, relacionadas con tres de los objetivos ya indicados.

\section{Resultados.}

El anunciado envejecimiento de la población es hoy una realidad y, con ella, los conceptos de envejecimiento activo (Active aging) y formación permanente (Lifelong learning) han adquirido un protagonismo creciente dentro de las políticas de empleo de la Unión Europea.

El primero de ellos surge como consecuencia de las nuevas circunstancias socio-económicas que propiciaron que las instituciones europeas asumieran la estrategia del envejecimiento activo e incorporaran el concepto en sus Directrices de los años 1999 y 2000 (Consejo Europeo, 2000a) y en los posteriores encuentros celebrados por el Consejo Europeo como la Estrategia de Lisboa del año 2000, el Consejo Europeo de Estocolmo en 2001 y el Consejo Europeo de Barcelona en 2002 (Consejo Europeo, 2000b, 2001 y 2002). Estos encuentros permitieron establecer acuerdos en torno al nuevo concepto de envejecimiento activo previamente delimitado por la Organización Mundial de la Salud (OMS, 2000) como un proceso de optimización de oportunidades de salud, participación y seguridad con el objetivo de mejorar la calidad de vida de las personas a medida que éstas envejecen. El concepto se asienta en pilares básicos como la salud, la independencia, la productividad y la protección a fin de aumentar la calidad de vida en la vejez mediante hábitos de vida saludables y una prospectiva vital confortable en esta nueva etapa. El foco de atención se desplaza de las personas mayores, como grupo separado, a todos los ciudadanos dado que el envejecimiento es un proceso presente en todas las personas (OCDE, 2006). A partir de 2001 se otorgó una mayor relevancia al envejecimiento activo convirtiéndolo en una directriz independiente que reclamaba acciones para propiciar: por un lado, los necesarios y profundos cambios en las actitudes sociales predominantes que existían hacia los trabajadores de más edad, buscando sensibilizar a los empresarios sobre el potencial de dichos trabajadores; y, por otro lado, la revisión de las ventajosas medidas fiscales que fomentaban la desmotivación frente al trabajo para ofrecerles, a cambio, otras que complacieran sus expectativas y les animasen a permanecer dentro del mercado de trabajo (Hessel, 2008).

En relación al segundo de los conceptos señalados, el enfoque de la formación permanente, también denominado Aprendizaje a lo largo de la vida (Lifelong learning), representa otra de las piezas clave de la economía global y de la sociedad de la comunicación y del conocimiento. Tiene su origen en el encuentro celebrado en Roma en 
el año 1994 por The Euopean Lifelong Learning Initiative (ELLI) (Candy, 1996) al referirse al potencial humano de las personas a través de un proceso de apoyo persistente en el tiempo que estimule y capacite a los sujetos para adquirir conocimientos, valores, habilidades y comprensión de las cosas que van a necesitar, y para saber aplicarlos con confianza, creatividad y satisfacción en todos aquellos roles, circunstancias y ambientes en los que se vean involucrados a lo largo de toda su vida. Subyace así un interés especial por dotar a los ciudadanos de las herramientas que les permitan adquirir la capacidad de «aprender a aprender» para que puedan adaptarse con eficacia a los cambios que se producen en nuestras comunidades sociales. El término se asocia a la actitud de compromiso que los trabajadores deben adoptar para mantenerse en constante ejercitación y desarrollarse en todas sus potencialidades. El objetivo radica en conseguir que las personas continúen formándose no solo para superar etapas académicas, o adquirir títulos, sino para ser capaces de gestionar su propio aprendizaje (Empowering individuals), para reforzar su autoestima y alcanzar la plena satisfacción personal (Del Prete, Gisbert \& Camacho, 2013). Existen tres razones básicas que justifican la necesidad de incrementar la formación de los trabajadores, especialmente, la de aquellos que llevan desempleados más de un año y que cuentan con una baja cualificación (García, Casal, Merino \& Sánchez, 2013):

a) la relevancia de obtener una formación inicial sólida sobre la que asentar futuros aprendizajes y procesos de formación continua;

b) los vertiginosos cambios económicos que inciden en los patrones productivos e impiden respuestas rápidas y eficaces ante las demandas del mercado laboral, y

c) la competitividad económica que exige una mano de obra cada vez más formada y especializada, capaz de acomodarse a los cambios con rapidez y eficacia.

Las políticas desarrolladas bajo el modelo del envejecimiento activo tratan, por lo tanto, de potenciar las capacidades de las personas cuando envejecen mediante la formación para que puedan desarrollar una vida productiva en la sociedad haciéndoles formar parte de la economía. El término activo se refiere, así, a la participación en asuntos sociales, económicos, culturales, espirituales y cívicos, y no solo a estar activos física o económicamente.

\subsection{Características socio-laborales de los trabajadores y trabajadoras mayores en España.}

Las personas mayores de 65 años representan en la actualidad, aproximadamente, el 17\% de la población activa de la UE y las estimaciones para el año 2050 auguran una subida que alcanza el 28,61\%, según la Agencia Euroestat (2013). En nuestro país, el Instituto Nacional de Estadística señalaba, a 1 de julio de 2013, un porcentaje del $17,9 \%$ de la población total (46.609.652 personas) de las que 3.580.971 eran hombres y 4.763 .975 mujeres; estimando para el año 2050 que la población de personas mayores en España de 65 años y más años superará ampliamente los 15,22 millones (un $36,38 \%$ sobre una población total de 41.863.016 personas). Esto quiere decir que más de la tercera parte de la sociedad española estará compuesta por personas mayores de 65 años, a las que les une exclusivamente la edad que comparten, ya que 
si algo caracteriza a este grupo poblacional es su heterogeneidad. Y es que existen notables diferencias entre ellas en función de su esperanza media de vida, hábitat, renta, cultura, estado civil, estructura familiar, condiciones bio-psicológicas, salud, formación, intereses, etc. Dentro de este colectivo, se integran los trabajadores mayores (Longer worker) que, según el Comité de la Naciones del año 2003, se compone de hombres y mujeres con edades comprendidas entre los 45 a los 64 años. En España los principios del envejecimiento activo se recogen en la legislación mediante la Ley 56/2003, de 16 de diciembre, de Empleo y en sus sucesivas reformas, destacando entre ellas la realizada en el año 2012 con el Real Decreto-ley 3/2012, de 10 de febrero, de medidas urgentes para la reforma del mercado laboral, que se inscribe dentro las iniciativas propuestas con la Declaración de la Unión Europea del año 2012 como «Año Europeo del Envejecimiento Activo y de la Solidaridad Intergeneracional» del que emanaron varios documentos encaminados a impulsar el envejecimiento activo en todas las facetas de la vida, incluyéndose la vertiente profesional, familiar y de la salud (Gómez, en prensa). Según dicha Declaración, el envejecimiento activo y la solidaridad intergeneracional requieren, entre otros aspectos, los siguientes:

a) La participación a lo largo de la vida de cada persona de forma que se aseguren las oportunidades y el acceso a los servicios, así como a las actividades políticas, sociales, recreativas y culturales; y el voluntariado que ayuda a mantener los lazos sociales y evitar el aislamiento.

b) El fomento de la participación en el mercado de trabajo mediante medidas en pro de la participación de los jóvenes y de los mayores en las actividades de formación y de aprendizaje permanente.

c) El reconocimiento de los valores de cada grupo de edad y de su contribución a la sociedad.

d) El fomento de la participación en la sociedad para vivir más tiempo y con mayor independencia.

e) El fomento de la salud, la prevención y el diagnóstico precoz de enfermedades a lo largo de todo el ciclo vital, así como la rehabilitación, que conduzcan a un envejecimiento activo, sano y a una vida independiente, al mismo tiempo que se tienen en consideración las diferentes necesidades de las mujeres y de los hombres.

f) La adaptación de los sistemas de seguridad social para que permitan ofrecer pensiones sostenibles y adecuadas para contribuir a reducir el número de personas mayores -con especial atención a las mujeresque subsisten por debajo del umbral de la pobreza permitiéndoles vivir con dignidad.

Expuestas las bases definitorias de la cultura del aprendizaje permanente, y a la luz de los datos ofrecidos por la Encuesta de Población Activa (INE, 2014) para el segundo trimestre del año 2014, solo puede decirse que en España la adopción de la cultura del aprendizaje a lo largo de la vida está lejos de convertirse en una realidad. Los datos muestran que entre las personas activas con edades comprendidas entre los 55 y más años una abrumadora mayoría no cursan estudios. De aquellas que sí cursan estudios la gran mayoría opta por realizar solo estudios en la enseñanza no reglada, seguidas de las que cursan estudios solo reglados y, por último, en menor número las personas que cursan estudios de las dos modalidades. En cuanto al género no existen diferencias con la tendencia general que se acaba de exponer. 
Analizando detenidamente la presencia de personas activas en ambas modalidades de formación, en cuanto a los estudios reglados se demandan especialmente estudios de Educación Superior, seguidos de los estudios de Bachillerato y de los estudios de Formación Profesional de Grado Medio, siendo igual la tendencia para ambos géneros. Respecto a los estudios no reglados la tendencia es exactamente la misma que en el caso de la enseñanza reglada, a diferencia de que el número de personas es mucho mayor en los cursos no reglados. Los mismos datos muestran resultados similares, 183.000 personas cursaron estudios no reglados, frente a las 10.000 personas que lo hicieron en la enseñanza reglada. Por sexos, en un total de 106.300 mujeres optaron por la enseñanza no reglada, frente a los 76.700 hombres que se decantaron por esta opción. En el caso de las personas inactivas entre 55 y 69 años, tanto en el segundo trimestre del año 2012 como en el 2014, es mayor el número de personas que no cursa estudios frente a aquellas que sí lo hacen y, respecto a estas últimas, es significativo que cursen estudios las personas de más edad, es decir, las de edades comprendidas entre 65 y 69 años, cursando estudios muchas más mujeres que hombres, con el predominio los estudios no reglados frente a los reglados.

\subsection{Características del contexto socio- laboral desde una perspectiva histórica.}

Hasta finales de los años 90 del pasado siglo las políticas de empleo desarrolladas en el marco de la UE persiguieron, básicamente, allanar el camino a la jubilación de los trabajadores mayores con el fin de favorecer el cambio generacional y hacer frente al desempleo juvenil. Lejos de conseguir este propósito los efectos de estas políticas han puesto en grave riesgo los sistemas de pensiones de los países de la UE, sin que el desempleo entre los jóvenes haya dejado de ser un problema cada vez más importante. Ahora se hace imprescindible fomentar la permanencia en el empleo de los trabajadores de más edad, los integrados en las cohortes de edad que abarcan los 55 y más años, y las distintas resoluciones publicadas por el Consejo de la Unión Europea, a lo largo de las últimas décadas, constatan este giro en las políticas de empleo fuertemente influenciadas por la globalización económica y la crisis financiera actual.

En consonancia con la Estrategia Europea de Empleo, y con las orientaciones generales de política económica de la Estrategia de Lisboa (2000), la Comisión Europea (2004) propuso que los Estados miembros elaborasen estrategias globales de envejecimiento activo que integrasen los siguientes elementos:

Reformar las disposiciones sobre jubilación anticipada a fin de lograr que resulte más rentable permanecer en el mercado de trabajo que abandonarlo vía jubilación.

Garantizar buenas condiciones de seguridad y salud laboral para lograr el bienestar de los trabajadores de más edad y sus posibilidades de seguir en el mercado laboral.

- Fomentar las formas flexibles de organización del trabajo entendiendo que podían favorecer el mantenimiento en activo de los trabajadores de más edad. A este respecto se apuntaban como fórmulas indicadas el empleo parcial que podría compaginarse con una jubilación parcial o con un empleo por cuenta propia.

Desarrollar estrategias de formación y de aprendizaje permanente de la población 
de mayor edad al entender que constituían una oportunidad para que reforzasen y actualizasen sus conocimientos y desarrollasen las capacidades necesarias para aprovechar plenamente las tecnologías de la información y comunicación.

En un panorama como el que se acaba de describir España se suma a la iniciativa en curso promovida por la Comisión Europea denominada «Estrategia Europa 2020» (Comisión Europea, 2010); una estrategia acordada por la Comisión Europea que pretende cinco objetivos en materia de empleo, innovación, educación, integración social y clima/energía para ser desarrollados en un plazo de 10 años: garantizar el empleo al 75\% de las personas de 20 a 64 años; invertir el 3\% del PIB de la UE en investigación y desarrollo; situar las emisiones de gases de efecto invernadero un 20\% (o incluso un 30\%) por debajo de los niveles de 1990, generar el $20 \%$ de nuestras necesidades de energía a partir de fuentes renovables y aumentar la eficacia energética un 20\%; reducir las tasas de abandono escolar por debajo del 10\% y lograr que al menos un $40 \%$ de las personas de 30 a 34 años hayan terminado estudios superiores y reducir al menos en 20 millones el número de personas en riesgo de pobreza o exclusión social.

Estos objetivos representan metas comunes para todos países miembros de la UE a los que se insta a desarrollar acciones tanto a nivel nacional como a nivel comunitario, ya que conforman los parámetros definitorios de lo que la propia UE espera llegar a ser en el año 2020. La Comisión Europea (2013) incide en la importancia de adquirir un compromiso serio con el desarrollo de las medidas en el ámbito de competencias nacionales en las que se combinen los tres enfoques básicos que sustentan la estructura de la Estrategia Europa 2020: un crecimiento inteligente basado en la apuesta por el conocimiento y la innovación; un crecimiento sostenible basado en el uso eficaz de los recursos y orientado hacia las energías limpias y, por último, en un crecimiento integrador que ofrezca numerosas opciones de empleo, en una economía que respalde la cohesión social y territorial.

\subsubsection{Valoración del impacto de la} iniciativa española frente a la crisis de empleo de los trabajadores de 55 y más años deedad.

Por lo general, las recomendaciones e iniciativas europeas dirigidas específicamente a los trabajadores mayores han estado siempre solapadas dentro de otras más amplias que las englobaban, a la espera de que se concretasen en propuestas específicas iniciadas por los gobiernos nacionales. En este marco surge la «Estrategia 55 y más» (E55+) como fruto del compromiso adoptado en el Acuerdo Social y Económico (ASE) firmado el 2 de febrero de 2011 entre el Gobierno y las organizaciones sindicales. Planteada con una vigencia de dos años, de 2012 a 2014, la Estrategia española incluye medidas en materia de empleo, formación y condiciones de trabajo con el objetivo de conseguir la prolongación de la vida laboral entre los trabajadores mayores de 55 años en desempleo. El diseño de la Estrategia se sustenta en la adopción de una perspectiva integral por parte de las administraciones públicas que facilite el acceso a los fondos necesarios y a los incentivos económicos asociados a los programas de formación en empresas dependientes de los Servicios Públicos de Empleo (SEPE) convocantes de 
las ofertas de la Formación Profesional para el Empleo.

La E55+ se regula mediante la Resolución de 14 de noviembre de 2011 por la que se publica el Acuerdo de Consejo de Ministros de 28 de octubre de 2011 que, a su vez, aprueba la «Estrategia Global para el Empleo de los Trabajadores y Trabajadoras de Más Edad 2012-2014», nacida como un instrumento para establecer el marco general de las políticas que se dirijan a favorecer el empleo de las personas de más edad, considerándose como tales a los trabajadores y a las trabajadoras de más de 55 años, sin perjuicio de que algunas de las medidas propuestas puedan ser aplicadas a personas de 45 años en adelante. La norma incide en la situación de las mujeres desempleadas pertenecientes a este rango de edad cuya tasa sigue aumentando en mayor medida que los hombres, aunque ambos casos comparten una situación peor cuanto menor es su cualificación profesional.

Los objetivos generales que orientan la E55+ son los siguientes:

a) Elevar la tasa empleo y reducir el desempleo de los trabajadores y las trabajadoras mayores de 55 años.

b) Favorecer el mantenimiento en el empleo de los trabajadores y trabajadoras mayores de 55 años para contribuir a la prolongación de la vida laboral y a la existencia de carreras de cotización a la Seguridad Social más largas.

c) Mejorar las condiciones de trabajo de los trabajadores y las trabajadoras mayores de 55 años, con particular atención a la mejora de su seguridad y salud en el trabajo.

d) Promover la reincorporación al mercado de trabajo de los trabajadores y las trabajadoras de más edad que pierden su empleo, asegurando una adecuada protección social durante su permanencia en situación de desempleo.

Las medidas que contiene la Estrategia española se distribuyen en tres categorías, pero solo en la segunda de ellas se hace referencia a medidas de carácter formativo. En todos los casos, cabe destacar la vaguedad e imprecisión con las que se expresan las medidas presentadas que se articulan en torno a varias líneas de actuación que han de servir para potenciar un tratamiento específico por parte de las Administraciones Públicas y de los interlocutores sociales de la situación de las personas mayores de 55 años desempleadas. La Estrategia española pretende ofrecer una atención equiparable, en importancia e intensidad, a los esfuerzos que se realizan con otros colectivos afectados por el desempleo en el actual contexto económicolaboral marcado por la consolidación fiscal y la austeridad en el gasto público. Sin embargo, a estas alturas, puede decirse que la Estrategia española ha tenido una escasa repercusión social, y aún menos éxito, a la luz de los resultados obtenidos una vez finalizado su periodo de vigencia. Los datos ofrecidos por la INE (2014) muestran que la E55+ no ha sido capaz de alcanzar los objetivos previstos ya que, lejos de conseguir que el sector de los trabajadores mayores de 55 años alcance cotas más altas de empleo, desde que se inició el año 2012 hasta el final del pasado año 2014 el número de parados mayores de 55 años ha aumentado en 160.500 personas.

\subsection{El nivel de alfabetización digital como indicador de empleabilidad en la población trabajadora de mayor edad.}

Los trabajadores que desarrollan su labor en un entorno en el que se imparte formación, 
así como aquellos que trabajan en sectores profesionales que requieren un nivel de cualificación superior, suelen estar más involucrados en procesos de aprendizaje, ajuste y reciclaje demostrando una capacidad de aprendizaje prácticamente intacta a lo largo de su vida. Solo las enfermedades incapacitantes que merman las capacidades físicas y/o cognitivas de las personas pueden afectar a las estrategias y habilidades de aprendizaje ya adquiridas que les permiten seguir aprendiendo. La capacidad de aprendizaje de las personas no viene determinada por la edad (Rodríguez, 2007), aunque sí se encuentra supeditada a las condiciones de trabajo en las que se han desenvuelto los trabajadores a lo largo de su vida laboral. Todas las personas, a cualquier edad, «aprendemos a lo largo y ancho de nuestras vidas, pues cualquier entorno relacional o situación vivida nos proporciona experiencia de la que extraer aprendizajes» (Bermejo, 2013, p.105). De igual manera, el potencial productivo de las personas tampoco se ve afectado por el envejecimiento (Travieso \& Panella, 2008) pues la disminución del rendimiento que suele achacarse a la edad también podría deberse a otras circunstancias como la obsolescencia de las cualificaciones o a un fenómeno de desgaste (burn-out) que puede afectarnos a cualquier edad. Contra estos fenómenos se lucha mediante una formación acomodada a las necesidades de aprendizaje que se identifiquen y con la adaptación de las condiciones de trabajo.

Uno de los retos a los que se enfrentan las personas con edades superiores a los 55 años está en alcanzar el dominio de los lenguajes tecnológicos y audiovisuales propios de la sociedad de la información y el conocimiento: la alfabetización digital. Este concepto engloba un gran número de habilidades, conocimientos, actitudes y destrezas que se han convertido en esenciales para nuestro quehacer diario (gestionar nuestro dinero, comprar, identificarnos, relacionarnos, trabajar, etc.). La alfabetización digital implica mucho más que el manejo más o menos eficaz del ordenador pues supone la adopción de una actitud que posibilite el dominio de las ideas, la adquisición de una conciencia crítica, la apertura al mundo, la participación plena y responsable en la sociedad, etc. (Coll, Mauri \& Onrubia, 2008; Flecha \& Elboj, 2000; González, Fanjul \& Cabezuelo, 2015). Dominar el lenguaje digital se erige como una destreza básica asociada a la supervivencia personal, social y laboral con un objetivo básico: favorecer la calidad de vida de todas las personas, cualquiera que sea su condición, sexo, raza, edad, religión, origen, etc.

Inmersos como estamos en la complejidad del mundo actual nos percatamos de la necesidad de propiciar «una innovación social paralela» (Ortega, 2013, p.161) que sea la artífice del acceso real, y en igualdad de condiciones, a la amplia gama de ofertas tecnológicas disponibles para evitar la brecha digital en la que se alojan la mayor parte de las personas mayores de nuestro país y que se relaciona directamente con la situación económica del usuario, por lo que puede señalarse la relación directa entre pobreza y brecha digital (Barroso, Cabero \& Romero, 2002). Un fenómeno que afecta de manera especial a las mujeres por las siguientes razones (Defensor del Pueblo Andaluz, 2011):

Por su condición de persona mayor, ya que en este segmento de edad se establece una brecha digital más acusada que en otras cohortes de edad. Las razones suelen estar asociadas a temores y autocensuras que 
tienen su germen en un sentimiento de inseguridad derivado de las escasas oportunidades de experimentar con las TIC de las que han disfrutado en general, lo que les infunde temor a perder el control en el manejo del ordenador. Junto al miedo se sitúa la vergüenza de reconocer su falta de conocimiento y destrezas con las nuevas tecnologías, desbordándoles la ingente información que deben conocer pero que no logran dominar. Si a esto se le suma el hecho de desconocer las ventajas de la experimentación mediante el método de ensayo-error, y/o las malas experiencias previas de aprendizaje, nos encontramos con personas desmotivadas frente a los recursos y herramientas virtuales.

- Por su mayor esperanza de vida respecto a los hombres y, por tanto, su representación es mayor dentro de esta franja de edad.

- Por encontrarse en una situación de mayor exclusión social dado el papel tradicional que han venido desarrollando tanto dentro del hogar como en la sociedad donde han realizando tareas de baja cualificación que no suelen requerir el uso de las TIC.

Las posibilidades que ofrecen las TIC para interactuar y relacionarse dentro de la sociedad de la información y el conocimiento garantiza a las personas mayores alcanzar cotas más altas de integración social, llegando a diluir los límites que tradicionalmente llevaba aparejada la edad. Actualmente, la vida laboral de los trabajadores y trabajadoras mayores está ligada al manejo y uso de las TIC como herramienta de trabajo, en mayor o menor medida en función del nivel de formación requerido para el desempeño de sus tareas profesionales y laborales. Esto ha propiciado que las TIC se hayan incorporado de manera natural al día a día de los trabajadores traspasando la frontera entre vida laboral y personal favoreciendo, así, que estos trabajadores continúen utilizándolas más allá de su vida activa.

\section{Reflexiones finales.}

Un factor esencial en la incorporación y uso de las TIC por parte de la población sea cual sea su edad, especialmente en el caso de las mujeres, es el nivel formativo alcanzado. Por este motivo las políticas de impulso y fomento de la inclusión digital han de adoptar una perspectiva transversal en el tratamiento de la igualdad de género que implique a todos los ámbitos, niveles y etapas en las que se vean involucradas las personas mayores. Su diseño debe basarse en la igualdad de mujeres y hombres en el acceso y el uso de las TIC lo que supone no solo potenciar el uso de las nuevas tecnologías por parte de este colectivo sino, también, atender al diseño mismo del hardware y del software para que se adapten a las personas y a sus circunstancias (Luna, Mendoza \& Álvarez, 2015). No debe olvidarse la falta de neutralidad de la investigación científica y tecnológica, y del desarrollo de las aplicaciones que emanan de ellas, cuya intencionalidad se centra en satisfacer principalmente las necesidades de un mercado más joven y ferviente consumidor de sus productos, antes que hacerlo asequibles a un sector de la población mayoritariamente sumido en una profunda brecha digital.

Las herramientas actuales de formación como la modalidad de teleformación y la modalidad e-learning ofrecen ventajas que los mayores no serán capaces de apreciar si no se sienten capaces y motivados para 
emprender el reto de seguir formándose en y mediante las TIC. Corresponde a las Administraciones públicas adoptar la perspectiva de la transversalidad para cerrar la persistente brecha digital que existe, con especial profundidad, en el segmento de los trabajadores mayores; puesto que la inclusión digital de los más jóvenes puede decirse que se hace cada día más real.

Por último, la investigación acerca del aprendizaje permanente orientado a los trabajadores de más edad requiere un impulso acorde con el papel fundamental que juega en la lucha frente al devenir del mercado laboral presente y futuro. La sociedad del conocimiento exige una formación dirigida hacia procesos más densos en conocimiento, por lo que la investigación puede ofrecer más información sobre cómo estos procesos afectan a los trabajadores y trabajadoras de diferentes segmentos de la población, incrementando las opciones de adaptación de los programas de formación a las características individuales de los trabajadores.

\section{Fuentes de financiación.}

El Proyecto DER 2012-36755. «Mercado de trabajo, transiciones laborales y edad: jóvenes y mayores de años» está financiado por la Secretaría de Estado de Investigación, Desarrollo e Innovación, dependiente del Ministerio de Economía y Competencia, y pertenece a los proyectos concedidos en la convocatoria del año 2012 del subprograma de Proyectos de Investigación Fundamental no Orientada/LIA del Proyectos I+D+i.

\section{Referencias bibliográficas.}

Barroso, J., Cabero, J. \& Romero, R. (2002). Las personas mayores y las nuevas tecnologías: una acción en la sociedad de la información. Innovación Educativa, 12, 219337.

Bermejo, L. (2013). Aprendizaje a lo largo de toda la vida: los programas socioeducativos con personas mayores. En M. J. Gómez Torres (Ed). La atención a las personas mayores desde una aproximación multidisciplinar (pp.103-126). Barcelona: Horsori.

Blanco, H. (2012). Análisis de datos cualitativos y Atlas-ti: una experiencia de formación. Revista Digital Universitaria: Docencia, Investigación e Innovación, 1 (1), 103-114.

Candy, P. C. (1996). Promoting lifelong learning : Academic developers and the university as a learning organization. International Journal for Academic Development 1 (1), 7-18. doi: 10.1080/ 1360144960010102.

Coll, C., Mauri, M.T. \& Onrubia, J. (2008). Análisis de los usos reales de las TIC en contextos educativos: una aproximación socio-cultural. Revista Electrónica de Investigación Educativa, 10, 1. Recuperado de http://www.scielo.org.mx/ s c i e l o.p h p ? p i d = S 1607 40412008000100001\&script=sci_arttext.

Comisión Europea (2004). Aumentar el empleo de los trabajadores de más edad y retrasar su salida del mercado de trabajo, COM (2004) 146 final, 3 de marzo de 2004.

Comisión Europea (2010). Comunicación de la Comisión Europea, Europa 2020: Una estrategia para el crecimiento inteligente, sostenible e integrador, COM (2010) 2020, de 3 de marzo. Recuperado de: http:// 
e c. e u r o p a. e u / e u $2020 / \mathrm{pdf} /$ COMPLET\%20EN\%20BARROSO\%20 \%20\%20007\%20\%20Europe\%202020\%20\%20EN\%20version.pdf

Comisión Europea (2013). Europa 2020: la estrategia europea de crecimiento, Bruselas, 2013. Recuperado de: http:// ec.europa.eu/europe2020/index_es.htm

Consejo de la Unión Europea (2000a). Directrices para las políticas de Empleo de los países Miembros para el año 2000. Revista del Ministerio de Trabajo, 32, 235-242.

Consejo Europeo (2000b). Consejo Europeo de Lisboa. Conclusiones de la Presidencia. Bruselas: Parlamento Europeo. Recuperado de: http://www.europarl.europa.eu/summits/ lis1_es.htm

Consejo Europeo (2001). Consejo Europeo de Laeken (Estocolmo). Conclusiones de la Presidencia. Bruselas: Parlamento Europeo. Recuperado: de: http:// www.europarl.europa.eu/summits/pdf/ lae_es.pdf

Consejo Europeo (2002). Consejo Europeo de Barcelona. Conclusiones de la Presidencia. Bruselas: Parlamento Europeo. Recuperado de: http://www.europarl.europa.eu/bulletins/ pdf/01s2002_es.pdf

Defensor del Pueblo Andaluz (2011). Las personas mayores y las Tecnologías de la Información y de la Comunicación (TIC) en Andalucía, Informe final al Parlamento, 2011, 42-43. Recuperado de:

http://www.defensordelpuebloandaluz.es/ $\mathrm{s}$ i t e s/d e f a u l t / f i l e s/ PERSONAS\%20MAYORES\%20marcadores.pdf

Del Prete, A., Gisbert, M. \& Camacho, M.M. (2013). Las tic como herramienta de empoderamiento para el colectivo de mujeres mayores. El caso de la comarca del montsià (Cataluña). Píxel-Bit. Revista de Medios y
Educación, 43, 37-50. doi: http://dx.doi.org/ 10.12795/pixelbit.2013.i43.03

EPA (2014). Encuesta de Población Activa: Segundo Trimestre del año 2014. Madrid: Instituto Nacional de Estadística. Recuperado de http://www.ine.es/dyngs/INEbase/es/ operacion.htm?c=Estadistica_C\&cid=125473617 6918\&menu=resultados\&idp=1254735976595

Euroestat (2013). European Social Stadistics, 2013 Edition. Belgium. (Euroestat, pro_10c2150p). Recuperado de: http:// ec.europa.eu/eurostat/documents/3930297/ 5968986/KS-FP-13-001-EN.PDF/6952d8367125-4ff5-a153-6ab1778bd4da

Flecha, R, \& Elboj, C. (2000). La educación de las personas adultas en la sociedad de la información. Revista Educación XX1, 3, 141162. Doi: 10.59.44/educxx1.3.1.407.

García, M., Casal, J., Merino, R. \& Sánchez, A. (2013). Itinerarios de abandono escolar y transiciones tras la enseñanza secundaria obligatoria. Revista de Educación, 361, 361135. doi: 10.4438/1988-592X-0034-8082-RE.

Gómez, M. J. (en prensa). Inclusión social y personas de edad avanzada: la formación como activador de las transiciones laborales de los colectivos más desfavorecidos. En J.M. Ortega (Coord), Personas de edad avanzada y mercado de trabajo: entre el envejecimiento activo y la estabilidad presupuestaria. Murcia: Bomarzo.

Gómez, M. J. \& Ordóñez, R. (2014). Las transiciones de la formación al trabajo de los jóvenes españoles en el marco de las medidas educativas contempladas en la Estrategia de Emprendimiento y Empleo Joven, 2013/2016. Temas Laborales. Revista Andaluza de Trabajo y Bienestar Social, 127 (4), 177-217. González, C., Fanjul, C. \& Cabezuelo, F. (2015). Uso, consumo y conocimiento de las nuevas tecnologías en personas mayores en 
Francia, Reino Unido y España. Comunicar, 45, 23, 19-28. Doi: 10.3916/C45-2015-02.

Hessel, R. (2008). Envejecimiento activo en una sociedad de personas mayores: formación para todas las edades. Revista Europea de Formación Profesional, 45 (3), 158-180.

«Ley 56/2003, de 16 de diciembre, de Empleo». Boletín Oficial del Estado $n^{\circ} 301$ (17de diciembre de 2003), págs. 44763-44771.

INE (2014). Encuesta de Población Activa: Segundo Trimestre del año 2014. Madrid: Instituto Nacional de Estadística. Recuperado de http://www.ine.es/dyngs/ INEbase/es/operacion.htm?c=Estadi stica_C\&cid=1254736176918\&m enu=resultados\&idp=1254735976595

López, F. (2002). El análisis de contenido como método de investigación. XXI Revista de Educación, 4, 167-179.

Luna, H., Mendoza, R. \& Álvarez, F.J. (2015). Patrones de diseño para mejorar la accesibilidad y uso de las aplicaciones sociales para adultos mayores. Comunicar, 45, 23, 85-94. Doi: 10.3916/C45-2015-09.

Marhuenda, F., Bernard, J.C. \& Navas, A. (2010). Las prácticas en empresa como estrategia de enseñanza e inserción laboral: las empresas de inserción social. Revista de Educación, 351,139-161. doi: 10.4438/1988592X-0034-8082-RE.

OCDE (2006). Older Workers. Living longer, working longer. Boletín DELSA, 2006, $n^{\circ} 2$. Recuperado de: http://www.oecd.org/social/ family/35961390.pdf

OMS (2000). Envejecimiento activo: un marco político, Organización Mundial de la Salud. Recuperado de: http:// envejecimiento.csic.es/documentos/ documentos/oms-envejecimiento-01.pdf

Ortega, J. M. (2013). Los mayores ante las Tecnologías de la Información y la
Comunicación. En M.J. Gómez Torres (Ed), La atención a las personas mayores desde una aproximación multidisciplinar (149174). Barcelona: Horsori.

Outón, P. \& Suárez, A. (2010). Adaptación y validación del test de dislexia Bangor. Revista de Investigación Educativa, 28 (2), 445-457.

«Real Decreto-ley 3/2012, de 10 de febrero, de medidas urgentes para la reforma del mercado laboral». Boletín Oficial del Estado (11 de febrero de 2012), págs.12483-12546.

Rodríguez, R. (2007). Personas mayores y Aprendizaje a lo largo de la vida de las Tecnologías de la Información y la Comunicación. Píxel-Bit. Revista de Medios y Educación, 30. Recuperado de : http:// www.sav.us.es/pixelbit/pixelbit/articulos/n30/ n30art/art305.htm..

Travieso, J.L. \& Panella, J. (2008). La alfabetización digital como factor de inclusión social: una mirada crítica. UOCpapers. Revista sobre la sociedad del conocimiento, 6. Recuperado de: http://www.uoc.edu/ uocpapers/6/dt/esp/travieso_planella.pdf

Fecha de recepción: 04-06-2015

Fecha de evaluación: 16-06-2015

Fecha de aceptación: 09-07-2015 